\title{
On the excess of ultra-high energy cosmic rays in the direction of Centaurus A
}

\author{
Ruo-Yu Liu ${ }^{1,2}$, Xiang-Yu Wang ${ }^{1,2}$, Wei Wang ${ }^{3}$ and Andrew M. Taylor ${ }^{4}$
}

\begin{abstract}
A posteriori anisotropy study of ultra-high energy cosmic rays (UHECRs) with the Pierre Auger Observatory (PAO) has shown evidence of excess of cosmic ray particles above $55 \mathrm{EeV}$ within $18^{\circ}$ of the direction of the radio galaxy Centaurus A. However, the origin of the excess remains elusive. We simulate the propagation of different species of particles coming from the direction of Centaurus A in the Galactic magnetic fields, and find that only particles of nuclear charge $Z \lesssim 10$ can avoid being deflected outside of the $18^{\circ}$ window of Centaurus A. On the other hand, considering the increasingly heavy composition of UHECRs at the highest energies measured by PAO, a plausible scenario for cosmic rays from the direction of Centaurus A can be found if they consist of intermediate-mass nuclei. The chemical composition of cosmic rays can be further constrained by lower-energy cosmic rays of the same rigidity. We find that cosmic ray acceleration in the lobes of Centaurus A is not favored, while acceleration in the stellar winds that are rich in intermediate-mass nuclei, could meet the requirement. This suggests that the observed excess may originate from cosmic ray accelerators induced by stellar explosions in the star-forming regions of Centaurus A and/or the Centaurus cluster located behind Centaurus A.
\end{abstract}

Subject headings: cosmic rays

\section{Introduction}

Despite nearly one-century of effort, the origin of ultra-high energy cosmic rays (UHECRs) still remains unknown. It is believed that the detections of anisotropy in the arrival direction of UHECRs can provide a useful clue for recognising the sources. The Pierre Auger Observatory (PAO) has detected 69 comic rays with energy above $55 \mathrm{EeV}$ between January 2004 and December 2009. The correlating fraction of the arrival directions which are closer than $3.1^{\circ}$ from the position of an AGN within $75 \mathrm{Mpc}$ is $38 \%$ (i.e. 27 out 69 events), while the expected fraction is $21 \%$ for an isotropic distribution of these cosmic rays. The significance of the anisotropy has decreased compared to previous estimates with smaller datasets (The Pierre Auger Collaboration et al. 2007; The Pierre AUGER Collaboration et al. 2008, 2010). On the other hand, it is reported that 13 events are located within $18^{\circ}$ of Centaurus A (Cen A) out of a total of 69 events in the whole sky above $55 \mathrm{EeV}$, whereas 3.2 events would be expected from this region (The Pierre AUGER Collaboration et al. 2010), implying that the clustering is not a statistical accident.

It has long been proposed that AGNs could accelerate particles to ultra-high energies (e.g. Mannheim 1995; Boldt \& Ghosh 1999; Berezinsky et al. 2006; Dermer et al. 2009). Particularly, Cen A, as the nearest AGN ( $d \sim$ $3.8 \mathrm{Mpc}$, Harris et al. 2010), was suggested by various authors as one potential source of UHECRs (e.g. Cavallo 1978; Romero et al. 1996; Anchordoqui et al. 2001; Hardcastle et al. 2009; Gureev \& Troitsky 2010; Pe'er \& Loeb 2011).

\footnotetext{
${ }^{1}$ Department of Astronomy, Nanjing University, Nanjing, 210093, China; ryliu@nju.edu.cn; xywang@nju.edu.cn

${ }^{2}$ Key laboratory of Modern Astronomy and Astrophysics (Nanjing University), Ministry of Education, Nanjing 210093, China

${ }^{3}$ National Astronomical Observatories, Chinese Academy of Sciences, 20A Datun Road, Chaoyang District, Beijing 100012, China

${ }^{4}$ ISDC, Chemin d'Ecogia 16, Versoix, CH-1290, University of Geneva, Switzerland
} 
However, there are two issues remaining to be solved before concluding that these UHECRs are from nearby AGNs. First, most of the nearby correlating AGNs are subluminous, so they may not be powerful enough to accelerate protons to $10^{20} \mathrm{eV}$ (see e.g. Waxman 1995; Piran 2010; Waxman 2011, but see Pe'er \& Loeb 2011), requiring intermediate mass nuclei or heavy nuclei as the composition of UHECRs in order to reach these energies. The other problem is the influence of magnetic field on the trajectories of cosmic rays. Both extragalactic and Galactic magnetic fields (EGMF and GMF) can deflect UHECRs, preventing them from pointing back to their birth places when observed from Earth. So the apparent arrival direction is not necessarily the real direction of the source and this influence is more serious for heavier nuclei. Recent measurements of the maximum air shower elongations $<\mathrm{X}_{\max }>$ and their fluctuations $\operatorname{RMS}\left(<\mathrm{X}_{\max }>\right)$ by the PAO indicate that the cosmic ray spectrum is gradually dominated by intermediate or heavy mass nuclei up to $E \sim 4 \times 10^{19} \mathrm{eV}$ (Abraham et al. 2010), although this claim depends on the hadronic interaction model at high energies which is not well-known at present 2. Interestingly, Hooper \& Taylor (2010) find that good fits to the $<\mathrm{X}_{\max }>$ and $\mathrm{RMS}\left(<\mathrm{X}_{\max }>\right.$ ) data can be found for the case in which the sources accelerate primarily intermediate mass nuclei.

In this paper, we examine the effect of Galactic magnetic fields on the deflection of UHECRs of different species that originate from the direction of Cen A and study the constraints on the composition of the UHECRs imposed by the excess in the direction from Cen A. Since the EGMF is poorly known, its impacts on UHECR deflection is not well understood. However, in general it is found that the deflections by the EGMF tend to be along and within the cosmic large scale structure of the galaxy distribution (Giacinti et al. 2010). For these reasons, we neglect the effect of the EGMF in this work and only consider the effect of the GMF on the deflection of UHECRs.

The rest of the paper is organized as follows. In §2, we briefly introduce the GMF models employed in this paper, as well as our method for simulating the propagation of UHECRs. We show our results and their implications in $\S 3$. In $\S 4$, we further constrain the chemical composition of UHECRs based on our analysis of the lower-energy cosmic rays with the same rigidity. We discuss the possible sources of the excess and give our conclusion in $\$ 5$. Throughout the paper, we use $\mathrm{eV}$ as the unit of particle energy and use c.g.s units for other quantities and denote by $Q_{x}$ the value of the quantity $Q$ in units of $10^{x}$, unless specified otherwise.

\section{Simulating propagation of UHECRs in the GMF}

The Galactic magnetic fields are generally described to be composed of a regular component in the disk and a large-scale field in the halo. The previous models for the magnetic field in the disk are either axissymmetric (e.g. Stanev 1997) or bi-symmetric (e.g. Han et al. 1999; Tinvakov \& Tkachev 2002). However, neither of these simple models agrees very well with observations (Han et al. 2006). Thus, in the present work, we employ two updated models, one described in Giacinti et al. (2010), which is based on the model built by Prouza \& Šmída (2003) (hereafter the PS model), and another developed in Jiang et al. (2010) (hereafter the J model) respectively. Both of these models contain a disk component and a halo component which consists of a toroidal field and a poloidal field. The halo component in these two models are basically the same. The field configurations are based on the anti-symmetric rotation measure

\footnotetext{
${ }^{1}$ Due to lack of statistics, all the events with energy larger than $\sim 4 \times 10^{19} \mathrm{eV}$ are used in the last bin to get the composition. So we can not get any information of $X_{\max }$ and $R M S\left(<X_{\max }>\right.$ ) at the highest energy (i.e. $\leq 10^{20} \mathrm{eV}$ ). In this paper, we assume the tendency which is shown in the lower energy range continues to the highest energy.

${ }^{2}$ It should be noted here that observation of High Resolution Fly's Eye Experiment (HiRes) did not show any correlation between arrival direction of UHECRs and nearby AGNs, and observation of Telescope Array (TA) can not distinguish an isotropic distribution from an anisotropic distribution of UHECRs arrival directions in current statistics (Abbasi et al. 2004; Tsunesada et al. 2011). Additionally, both of their elongation measurements prefer a pure proton composition in high energy end(Abbasi et al. 2010; Tsunesada et al. 2011). Our paper is based on the observation of the PAO.
} 
(RM) sky revealed by the extragalactic radio sources (Han et al. 1997, 1999) and the vertical filaments in the Galactic center (Yusef-Zadeh et al. 1984, 2004), while their strengths are selected to meet the features of the observed filaments in the Galactic center (Morris \& Serabyn 1996) and the vertical field component in the vicinity of the Sun (Han \& Qiao 1994). For the disk component, there is a difference between these two models. In the PS model, the disk component is developed from a basic conservative model constructed by Han \& Qiao (1994), which was based on the Faradayrotation measurement of 134 pulsars, with the assumption of two logarithmic spiral arms and a constant pitch angle, showing bisymmetric (BSS) magnetic field (see Prouza \& Šmída 2003). While in the J model, the disk component is founded upon the RM of pulsars by Han et al. (2006), and adopts a four-arm spiral structure, whose configuration can be depicted by an Archimedes spiral (Hou et al. 2009), with the magnetic fields reversed from arms to inter-arms (Jiang et al. 2010; Nota \& Katgert 2010). We present the configurations of the disk magnetic field of these two models in Figure 1 to show the difference. A detailed description of both the PS model and the J model can be found in Giacinti et al. (2010) and Jiang et al. (2010) respectively.

Since the deflections of UHECRs caused by the small scale random component at the energies we consider are smaller or at most comparable to that induced by the regular GMF even for iron nuclei (Giacinti et al. 2010; Tinyakov \& Tkachev 2005), the random component can be ignored as far as only the largest deflection angle is concerned in the present study.

The propagation of cosmic ray particles can be described by

$$
\left\{\begin{array}{l}
d \boldsymbol{p} / d t=Z e v \times B \\
d \boldsymbol{x} / d t=\boldsymbol{v}
\end{array}\right.
$$

where $e$ is the charge of the electron and $c$ is the speed of light in the vacuum. $\boldsymbol{p}, \boldsymbol{v}$ and $\boldsymbol{x}$ are the momentum, velocity and the spatial coordinates of the particle respectively, and $\boldsymbol{B}$ is the magnetic field. Here we do not consider any changes of the particle during propagation, such as fragmentation or cooling processes. Following the method used in previous works (e.g. Stanev 1997; Harari et al. 1999; Tinyakov \& Tkachev 2002; Prouza \& Šmída 2003; Giacinti et al. 2010; Jiang et al. 2010), we propagate an anti-proton or anti-nucleus from the earth along certain direction, tracking it until it reaches the border of the Galaxy, and recording its position and velocity direction at that moment. A proton or a nucleus which enters the Galaxy at the same position and with the same direction of its velocity will travel back to the Earth along the same path. The spatial extent of cosmic rays emitted by Cen A is assumed to be disk-like, with a typical radius of $5^{\circ}$ on the celestial sphere. Thus, particles which enter the Galaxy with a direction closer than $5^{\circ}$ from the center of an object are regarded as coming from that object.

To study the influence of the GMF on the arrival directions of cosmic rays coming from the direction of Cen A, we isotropically propagate and backtrace $10^{6}$ particles with energies from $10^{19.75} \mathrm{eV}(\sim 55 \mathrm{EeV}$, the threshold energy for the clustering) to $10^{20.15} \mathrm{eV}$ ( $\sim 142 \mathrm{EeV}$, the highest energy the PAO ever detected) with an increment of $10^{0.05}$ in energy for different species of particles separately. There are two important parameters for each particle. One is the angle between the particle's arrival direction and the direction of Cen A, which we denote as $\theta$. The other one is the angle between the direction of the particle's velocity when it enters the Galaxy and the direction of Cen A, denoted by $\alpha$. Figure 2 and 3 show the Galactic coordinates of arrival directions of particles coming from Cen A (i.e. $\alpha<5^{\circ}$ ) for the two GMF models respectively. Each dot represents a single particle, and the different colors represent their energies. The black filled star is the location of Cen A $\left(l=309.52^{\circ}, b=19.42^{\circ}\right)$ and the black solid curve is the projection on the celestial sphere of a circle centered on Cen A with a radius of $18^{\circ}$. The small open circles represent the observed events and the size of the circles is proportional to their energies. The 13 magenta circles represent the events correlated with Cen A. One can see that, in both GMF models, as expected, the smaller the particle's rigidity (i.e. $E / Z$, where $E$ and $Z$ are the energy and the nuclear charge of the particle respectively), the larger the deviation from the original direction. For heavy nuclei, such as iron or calcium nuclei, the particles are severely deflected even at the highest energy, while for lighter nuclei such as proton or helium nuclei, the particles can reach the earth without 
being much deflected.

\section{Effects of GMF on the arrival directions of UHECRs and its implications}

The observed 13 cosmic ray particles from the direction of Cen A spans the energy range from $10^{19.75} \mathrm{eV}$ to $10^{19.95} \mathrm{eV}$. The influence of GMF on the arrival directions of cosmic ray particles of different energies can be studied quantitatively if their energy spectrum is given. We assume that these particles are accelerated in Cen A to a power-law distribution with index $s$, and keep the same spectrum whilst propagating to the border of the Galaxy, since the distance of Cen A to us is much shorter than the attenuation length. Through our simulation of the propagation of these particles in the GMF, we get a mock distribution of arrival directions for particles of different species and different energies. For convenient comparisons with observations, we define two parameters: one is $\zeta_{i}(E)$, defined as the number ratio of particles of species $i$ and energy $E$ that reach the Earth when the deflection by the GMF is considered to those that reach the Earth without considering the GMF deflection. This factor is also known as the magnetic lensing amplification

factor (see, e.g. Harari et al. 2000, 2002; Giacinti et al. 2010). Another parameter is $\eta_{i}(E)$, defined as the number ratio of particles that reach the Earth from a direction within $18^{\circ}$ of Cen A (i.e. number of particles with $\theta<18^{\circ}$ ) to those that reach the Earth from all directions. In the calculations, we consider the relative exposure of the PAO (Sommers 2001). The corresponding ratios that are integrated over energy from $10^{19.75} \mathrm{eV}$ to $10^{19.95} \mathrm{eV}$ (approximately the energy range of the observed 13 events) can be obtained by

$$
\bar{\zeta}_{i}=\frac{\int \zeta_{i}(E) \frac{d N_{i}}{d E} d E}{\int \frac{d N_{i}}{d E} d E}
$$

and

$$
\bar{\eta}_{i}=\frac{\int \zeta_{i}(E) \eta_{i}(E) \frac{d N_{i}}{d E} d E}{\int \zeta_{i}(E) \frac{d N_{i}}{d E} d E} .
$$

Table 1 lists the values of these two ratios for different particle species for both the PS model and the J model. From the values of $\bar{\zeta}_{i}$, one can find that the flux of light particles are barely influenced by the GMF due to their high rigidity. Here, the flux of intermediate mass nuclei such as oxygen and silicon are magnified while that of heavy nuclei are slightly demagnified. The values of $\bar{\eta}_{i}$ are smaller as the nuclei get heavier, implying that heavy nuclei will be deflected severely from their original directions by the GMF.

Take iron nuclei as an example case. If the excess events from Cen A are dominated by iron nuclei, the allsky event number of iron nuclei contributed by Cen A should be $13 / \bar{\eta}_{\mathrm{Fe}}=1300$ for the PS model, far more than the observed event number which is 64 in the same energy range. On the other hand, it has been shown that it is highly unlikely for all the observed events with energy $>55 \mathrm{EeV}$ to come solely from a single source, because simulations of the arrival directions for such a case indicate that it would display an apparently dipolar pattern, which is different from the observed distribution even in the most favorable cases (Giacinti et al. 2011). Thus, we draw a circle centered at Cen A with a radius equal to the largest deflection angle (the pale solid circle in Figure 2 and 3), and regard the observed event number in the same energy range that the circle covers as the maximum event number $\left(N_{\max , i}\right)$ that Cen A can contribute to. For instance, for iron nuclei in the PS model, the maximum event number is 33 . In some cases (e.g. for light nuclei), the pale circles shrink into the $18^{\circ}$ window of Cen A, and we set $N_{\max }$ to be 13 in such cases. For iron nuclei in the PS model, they could contribute at most $N_{\mathrm{CenA}, \mathrm{Fe}}=N_{\max , \mathrm{Fe}} \times \bar{\eta}_{\mathrm{Fe}}=0.33$ events in the $18^{\circ}$ window of Cen A. Thus, as mentioned above, we disfavor iron nuclei as the dominant composition of the excess events.

Similarly, we also calculate the maximum event number that other species of particles can contribute for both GMF models. The results are shown in Table 1. One can see that the observed excess events around Cen A can be 
reconciled to particles with $Z \lesssim 10$ in the both models. Since heavy nuclei such as iron will be deflected severely, deviating far from their original direction, they can be ruled out as the main composition of the excess events from Cen A. We note that the distribution of simulated particles within the $18^{\circ}$ window is concentrated in some regions when only the regular component of the GMF is considered, which is not as scattered as the observed distribution. Since we aim to obtain constraints on the composition of excess cosmic rays, for which only the largest deflection angle is concerned, modeling the actual distribution is beyond the present work. However, despite this, we do note that the incorporation of random components of the GMF and/or appropriate EGMF would likely wash out the distribution obtained in the present simulation, making it look more like the observed event distribution (e.g. Yüksel et al. 2012).

\section{Constraints on the source composition with lower-energy cosmic rays}

The shower profile of UHECRs measured by PAO implies that the average chemical composition of cosmic rays consists of heavy or intermediate-mass nuclei, most notably at the highest energy bin, as long as the properties of hadronic interactions do not change significantly at such high energies. If the measured all-sky-averaged composition roughly reflects the average composition of particles from the direction of Cen A, light particles, such as protons and helium nuclei, can not be the dominant component of these events. Such a heavy or intermediate-mass nuclei composition could be achieved only if 1) the accelerated material is rich in intermediate-mass or heavy elements; or 2) the acceleration ability of the source is limited so that only intermediate-mass or heavy nuclei can be accelerated to energies $>10^{19.75} \mathrm{EeV}$ (i.e., the source is not powerful enough to accelerate light particles to energies $>10^{19.75} \mathrm{EeV}$ ). In the latter scenario, the composition in the energy range from $10^{19.75} \mathrm{eV}$ to $10^{19.95} \mathrm{eV}$ is dominated by intermediatemass nuclei or heavy nuclei, regardless of the chemical composition of the accelerated material. However, one should be cautious of lower-energy lighter cosmic rays that have the same rigidity, as they will follow the same trajectories and could produce stronger anisotropy in the direction of Cen A at lower energies if their abundance is not low Lemoine \& Waxman 2009; The Pierre Auger Collaboration et al. 2011). We will show below that the chemical composition of the accelerated material in this scenario must satisfy some constraint as well.

A number of acceleration sites of Cen A, such as inner jets/lobes (see, e.g. Dermer et al. 2009; Rieger \& Aharonian 2009; Honda 2009), the north middle lobe (NML) (see, e.g. Romero et al. 1996) as well as the giant lobes (see, e.g. Hardcastle et al. 2009; O'Sullivan et al. 2009), have been suggested for UHECR 3. Although there are no precise measurements of the element abundance of these possible acceleration regions in Cen A, we can roughly estimate the abundance from various observations in these regions. In the inner lobe case, UHECRs could be accelerated by shocks driven by the inner lobes inflating into the interstellar medium (ISM). So the chemical composition of the accelerated UHECRs in this case should be similar to that of the ISM. Deep Chandra observations of some nearby gas-rich elliptical galaxies show a near-solar metallicity of ISM generally, except an unequivocally sub-solar oxygen abundance $\left(\lesssim 0.5 Z_{\odot}\right.$, see $\operatorname{Kim} 2012$, and reference therein). For the NML, the spectra of five associated X-ray knots can be well fitted by sub-solar element abundance (Kraft et al. 2009), implying a sub-solar metallicity for NML. As for the giant lobes, since they extend out to $600 \mathrm{kpc}$ from the center, the ambient medium should be intergalactic medium (IGM), so the chemical composition of accelerated UHECRs is probably identical to that of IGM, which is found to be sub-solar metallicity both observationally and theoretically (e.g. Richter et al. 2009; Barai et al. 2011). Here we assume the solar abundance composition for cosmic rays accelerated in these regions and adopt the solar element abundance reported in Lodders \& Palme (2009) in the following calculation. For simplicity, we attribute elements with $6<Z<10$ to

\footnotetext{
${ }^{3}$ The central black hole with a strong magnetic field has also been suggested as a possible accelerator (e.g. Neronov et al. 2009), though other works have highlighted that the maximum energy may be somewhat limited, even for the of case of nuclei, see e.g. (Lemoine \& Waxman 2009; Rieger \& Aharonian 2009). For this reason we do not consider it in the present work.
} 
oxygen, with $11 \leq Z<16$ to silicon, with $17 \leq Z<23$ to calcium, and with $23 \leq Z \leq 26$ to iron, and neglect heavier elements. Then the relative mass abundance is $M_{\mathrm{H}}: M_{\mathrm{He}}: M_{\mathrm{O}}: M_{\mathrm{Si}}: M_{\mathrm{Ca}}: M_{\mathrm{Fe}} \approx 60: 24: 1: 0.16: 0.014: 0.12$.

Since cosmic rays of solar abundance composition are dominated by light elements such as hydrogen and helium, the condition 1) can not be satisfied and hence the condition 2) should be taken into consideration. We assume that the maximum and minimum energies of particles for different species are rigidity-dependent (i.e. $E_{\max }, E_{\min } \propto Z$ ), and the spectrum for nuclei with nuclear charge $Z_{i}$ is described by

$$
d N_{i} / d E=f_{i} N_{0}\left(E / E_{0}\right)^{-s} \quad E_{i, \min }<E<E_{i, \max },
$$

where $f_{i}$ is the relative number abundance of element $i$ at a given energy E, and $N_{0}$ and $E_{0}$ are used for normalization. Above $E_{\max }$ or below $E_{\min }$, an abrupt cutoff in the spectrum is assumed. Thus, the relative number abundance $f_{i}$ relates to the mass abundance by

$$
M_{i}: M_{j}=\int_{E_{i, \text { min }}}^{E_{i, \text { max }}} A_{i} \frac{d N_{i}}{d E} d E: \int_{E_{j, \text { min }}}^{E_{j, \max }} A_{j} \frac{d N_{j}}{d E} d E=A_{i} Z_{i}^{1-s} f_{i}: A_{j} Z_{j}^{1-s} f_{j}(i, j=\mathrm{H}, \mathrm{He}, \mathrm{C}, \ldots) .
$$

For a mixed composition, the maximum number of events within $18^{\circ}$ of Cen $\mathrm{A}$ is given by

$$
N_{\text {CenA,mix }}=\frac{\sum_{i} \int_{10^{19.75} \mathrm{eV}}^{10^{19.95}} \zeta_{i}(E) \eta_{i}(E) \frac{d N_{i}}{d E} d E}{\sum_{i} \int_{10^{19.75} \mathrm{eV}}^{10^{19.95} \mathrm{eV}} \zeta_{i}(E) \frac{d N_{i}}{d E} d E} \times N_{\text {max }, \text { mix }}
$$

where $N_{\text {max,mix }}$ is the maximum event number that could be contributed by Cen A, as defined in $\S 4$, for such a mixed composition. The value of $N_{\text {max,mix }}$ can be taken as 30, since one can find from Figure 2 and Figure 3 that for most species this value is roughly $\lesssim 30$ (see discussion in Section 3). Similarly, for a mixed composition, the average atomic mass of cosmic ray events contributed by Cen A is

$$
<A>=\frac{\sum_{i} \int_{10^{19.75} \mathrm{eV}}^{10^{19.95}} A_{i} \zeta_{i}(E) \frac{d N_{i}}{d E} d E}{\sum_{i} \int_{10^{19.75} \mathrm{eV}}^{10^{19.95}} \zeta_{i}(E) \frac{d N_{i}}{d E} d E} .
$$

One can see that both $N_{\text {CenA,mix }}$ and $\langle A\rangle$ are related with the maximum acceleration energy $E_{i, \max }$ through the spectrum $d N_{i}(E) / d E$. As the maximum energy is rigidity-dependent, $E_{i, \max }=Z_{i} E_{p, \max }$, where $E_{p, \max }$ is the maximum accelerating energy for protons. Any appropriate value of $E_{p \text {,max }}$ should lead to $N_{\text {CenA,mix }} \gtrsim 10$ (assuming 3.2 out of 13 events are from isotropically distributed sources in this region). On the other hand, the fits to both $<X_{\max }>$ and RMS $<X_{\max }>$ have shown that the all-sky-averaged composition of arriving UHECRs may be nitrogen-like (Taylor 2011), if only a small spread in composition exists at energies $\sim 10^{19.5} \mathrm{eV} 4$. Under the assumption that the events contributed by Cen A have the same composition as the measured all-sky composition, we conservatively set $<A>\gtrsim 10$ to be another necessary condition.

Figure 4 shows the maximum number $N_{\text {CenA,mix }}$ and the average atomic mass $\langle A\rangle$ of cosmic rays from the direction of Cen $A$ as a function of $E_{p, \max }$ for solar abundance composition of cosmic rays. The red lines represent the results for the PS model of the GMF, while the blue ones represent the results for the J model. One can see that in both GMF models, low $E_{p, \text { max }}\left(\right.$ e.g. $\lesssim 10^{19} \mathrm{eV}$ ) can be excluded, because in this case only heavy nuclei can be accelerated up

\footnotetext{
${ }^{4}$ More generally speaking, it is difficult to explain $<X_{\max }>$ and $\operatorname{RMS}\left(<X_{\max }>\right)$ simultaneously by any composition because of the low value of the measured $\operatorname{RMS}\left(<X_{\max }>\right)$. Indeed, a larger $\operatorname{RMS}\left(<X_{\max }>\right)$ is predicted by transition models in which the composition changes from a light to a heavier composition, as is suggested by the trend in $\left\langle X_{\max }\right\rangle$ (see e.g. The Pierre Auger Collaboration et al. 2011; Ulrich et al. 2009).
} 
to the required energy, while for heavy nuclei, due to their low-rigidity, most of them are deflected into other directions or even can not reach the Earth. So $E_{p \text {, max }}$ should be large enough to allow at least intermediate-mass nuclei to be able to reach $10^{19.75} \mathrm{eV}$. On the other hand, because of the large abundance of hydrogen or helium elements relative to oxygen (and heavier elements), the average chemical composition will become too light once $E_{\mathrm{He}, \max }>10^{19.75} \mathrm{eV}$. As a consequence, we have the pale shaded region and the dark shaded region (including the pale one) representing the range of $E_{p \text {,max }}$ in which both requirements can be satisfied for the PS model and the J model respectively. Note that the limit of $E_{p, \max }$ obtained here is consistent with the theoretical acceleration limit of Cen A (Lemoine \& Waxman 2009; Piran 2010), although the acceleration ability of Cen A is still under much debate (Rieger \& Aharonian 2009; Pe'er \& Loeb 2011).

Lower-energy cosmic rays that have the same rigidity $(E / Z)$ will travel along the same paths. The chemical composition of cosmic rays can be constrained by requiring that they do not produce excess in cosmic-ray flux in the direction of Cen A and in the all-sky flux at corresponding energies. As shown above, in the viable range of $E_{p \text {,max }}$ obtained, the observed excess events from the direction of Cen A are very likely to be dominated by intermediate mass nuclei with $6 \lesssim Z \lesssim 10$, so we take oxygen nuclei as the dominant particles in the following calculation. Given that the ratio between the number of excess cosmic rays from the direction of Cen A and that of the whole sky is 10: 64 in the energy range from $10^{19.75} \mathrm{eV}$ to $10^{19.95} \mathrm{eV}$, cosmic rays originating from Cen A should account for a flux $F_{\mathrm{O}} \simeq 10 / 64 F^{\mathrm{ob}}\left(10^{19.75-19.95} \mathrm{eV}\right)$ within the $18^{\circ}$ window around Cen $\mathrm{A}$ and an all-sky flux of $F_{\mathrm{O}, \text { all }}=F_{\mathrm{O}} / \eta_{\mathrm{O}}$ in the same energy range, where $F^{\mathrm{ob}}\left(10^{19.75-19.95} \mathrm{eV}\right)=A_{\exp } \int_{10^{19.75} \mathrm{eV}}^{10^{19.95} \mathrm{eV}} \frac{d N^{\mathrm{ob}}}{d E} d E$ is the measured all-sky flux, $d N^{\mathrm{ob}} / d E$ is the measured differential flux, and $A_{\exp }$ is the total exposure of the PAO. So particles of species $i$ that have the same rigidity in the energy range from $\frac{Z_{i}}{Z_{0}} 10^{19.75} \mathrm{eV}$ to $\frac{Z_{i}}{Z_{0}} 10^{19.95} \mathrm{eV}$ should produce a flux of $F_{i}=\frac{f_{i}}{f_{0}}\left(\frac{Z_{i}}{Z_{\mathrm{O}}}\right)^{1-s} F_{\mathrm{O}}$ inside the $18^{\circ}$ window of Cen A and an all-sky flux of $F_{i, \text { all }}=\frac{f_{i}}{f_{0}}\left(\frac{Z_{i}}{Z_{0}}\right)^{1-s} F_{\mathrm{O}, \text { all }}$, respectively.

Since the search at lower energies has not revealed any significant anisotropy signals around the Cen A region (The Pierre Auger Collaboration et al. 2011), the flux $F_{i}$ of lower-energy cosmic rays should not be higher than the expected background flux, i.e.

$$
\frac{f_{i}}{f_{\mathrm{O}}}\left(\frac{Z_{i}}{Z_{\mathrm{O}}}\right)^{1-s} F_{\mathrm{O}} \lesssim x F^{\mathrm{ob}}\left[\frac{Z_{i}}{Z_{\mathrm{O}}}\left(10^{19.75}-10^{19.95}\right) \mathrm{eV}\right]
$$

where $x \simeq 0.0466$ is the fraction of the exposure of the PAO within the $18^{\circ}$ window of Cen A (The Pierre Auger Collaboration et al. 2011). It should be pointed out here that the above inequality is a conservative constraint, since the anisotropy signal can be fairly strong when the source counts are comparable to the background counts, especially if the number of background counts is large. Note that this method is only applicable to lower-energy, lighter cosmic rays.

One can also get constraints on the cosmic ray composition by requiring that lower-energy all-sky flux produced by sources in the direction of Cen A should be lower than the measured flux in the same energy range, i.e.

$$
\frac{f_{i}}{f_{\mathrm{O}}}\left(\frac{Z_{i}}{Z_{\mathrm{O}}}\right)^{1-s} F_{\mathrm{O}} / \eta_{\mathrm{O}} \lesssim F^{\mathrm{ob}}\left[\frac{Z_{i}}{Z_{\mathrm{O}}}\left(10^{19.75}-10^{19.95}\right) \mathrm{eV}\right] .
$$

The measured cosmic ray flux in the relevant range can be fitted by a broken power-law, given by Abraham et al. 2010)

$$
\frac{d N_{\mathrm{ob}}}{d E}=\left\{\begin{array}{l}
N_{\mathrm{b}}\left(\frac{E}{E_{\mathrm{b}}}\right)^{-p_{1}}, E<E_{\mathrm{b}} \\
N_{\mathrm{b}}\left(\frac{E}{E_{\mathrm{b}}}\right)^{-p_{2}}, E>E_{\mathrm{b}}
\end{array}\right.
$$

where $E_{\mathrm{b}}=10^{19.46} \mathrm{eV}$ is the break energy, $N_{\mathrm{b}}$ is the normalized factor, $p_{1}=2.6$ and $p_{2}=4.3$ are the power-law indexes 
of the two segment, respectively. Then from inequality $(8)$ we obtain

$$
f_{i} \lesssim f_{\mathrm{O}} \times 6.4 x\left(\frac{Z_{i}}{Z_{\mathrm{O}}}\right)^{s-p_{1}}\left(\frac{1-p_{2}}{1-p_{1}}\right)\left(\frac{E_{2}^{1-p_{1}}-E_{1}^{1-p_{1}}}{E_{2}^{1-p_{2}}-E_{1}^{1-p_{2}}}\right) E_{\mathrm{b}}^{p_{1}-p_{2}} \text { for lighter elements, }
$$

and from inequality (9) we obtain

$$
f_{i} \lesssim f_{\mathrm{O}} \times \begin{cases}6.4 \eta_{\mathrm{O}}\left(\frac{Z_{i}}{Z_{\mathrm{O}}}\right)^{s-p_{2}} & \text { for heavier elements } \\ 6.4 \eta_{\mathrm{O}}\left(\frac{Z_{i}}{Z_{\mathrm{O}}}\right)^{s-p_{1}}\left(\frac{1-p_{2}}{1-p_{1}}\right)\left(\frac{E_{2}^{1-p_{1}}-E_{1}^{1-p_{1}}}{E_{2}^{1-p_{2}}-E_{1}^{1-p_{2}}}\right) E_{\mathrm{b}}^{p_{1}-p_{2}} & \text { for lighter elements. }\end{cases}
$$

Here $E_{1}=10^{19.75} \mathrm{eV}$ and $E_{2}=10^{19.95} \mathrm{eV}$. One can find that for lighter elements, which method gives a stronger constraint depends only on the value of $x$ and $\eta_{\mathrm{O}}$. In the case discussed in the present work, $x \ll \eta_{\mathrm{O}}$, so the constraints from the $18^{\circ}$ window anisotropy are stronger. But for heavier elements, useful constraints only come from the latter method. Assuming $s=2$, inequality (11) and (12) give $f_{\mathrm{H}} \lesssim 4.4 f_{\mathrm{O}}, f_{\mathrm{He}} \lesssim 2.9 f_{\mathrm{O}}, f_{\mathrm{Si}} \lesssim 0.72 f_{\mathrm{O}}, f_{\mathrm{Ca}} \lesssim 0.31 f_{\mathrm{O}}$ and $f_{\mathrm{Fe}} \lesssim 0.17 f_{\mathrm{O}}$, corresponding to $M_{\mathrm{H}} \lesssim 2.2 M_{\mathrm{O}}, M_{\mathrm{He}} \lesssim 2.9 M_{\mathrm{O}}, M_{\mathrm{Si}} \lesssim 0.72 M_{\mathrm{O}}, M_{\mathrm{Ca}} \lesssim 0.31 M_{\mathrm{O}}, M_{\mathrm{Fe}} \lesssim 0.18 M_{\mathrm{O}}$ for the PS model; and $f_{\mathrm{H}} \lesssim 4.4 f_{\mathrm{O}}, f_{\mathrm{He}} \lesssim 2.9 f_{\mathrm{O}}, f_{\mathrm{Si}} \lesssim 1.2 f_{\mathrm{O}}, f_{\mathrm{Ca}} \lesssim 0.51 f_{\mathrm{O}}$ and $f_{\mathrm{Fe}} \lesssim 0.29 f_{\mathrm{O}}$, corresponding to $M_{\mathrm{H}} \lesssim 2.2 M_{\mathrm{O}}$, $M_{\mathrm{He}} \lesssim 2.9 M_{\mathrm{O}}, M_{\mathrm{Si}} \lesssim 1.2 M_{\mathrm{O}}, M_{\mathrm{Ca}} \lesssim 0.51 M_{\mathrm{O}}, M_{\mathrm{Fe}} \lesssim 0.31 M_{\mathrm{O}}$ for the $\mathrm{J}$ model. A larger power-law index $s$ will put a more stringent constraint on the relative abundance of lighter particles. One can find that, in both GMF models, constraints on heavier elements are easy to satisfy, i.e. the excess in flux at higher energy induced by heavier nuclei with the same rigidity can be avoided. But to avert a low energy excess in the Cen A direction made by protons and helium nuclei with the same rigidity, a super-solar metallicity especially a super-solar intermediate-mass element abundance is required. This result is consistent with the analysis from fitting $X_{\max }$ and $\operatorname{RMS}\left(<X_{\max }>\right.$ ) that measured by the $\mathrm{PAO}$ (Shaham \& Piran 2012). Such a chemical composition is not favored by the candidate cosmic-ray accelerators in the radio galaxy Cen A. In other words, if we attribute the 10 excess events to Cen A, a stronger excess would also occur at 1-10 EeV. We note, however, that if the composition of these 10 clustering events turns out to be proton or helium nuclei, different from the measured all-sky-averaged composition, then the above conclusion does not apply (for light nuclei composition, see e.g. Fargion 2011, and reference therein).

\section{Discussion and Conclusion}

The required chemical composition of the excess cosmic-rays in the direction of Cen A seems to disfavor the above mentioned candidate sites in Cen A. The requirement, however, can be more easily satisfied by UHECR accelerators induced by stellar explosions since either the stellar wind of massive stars or the exploded ejecta can be rich in intermediate mass nuclei. The chemical composition of WR stellar winds is $M_{\mathrm{He}}: M_{\mathrm{C}}: M_{\mathrm{O}}: M_{X}=0.32: 0.39: 0.25: 0.04$ (Bieging 1990; van der Hucht et al. 1986) where $M_{X}$ is the sum of elements heavier than oxygen, which we treat simply as silicon in the following calculation. The composition of the hypernova ejecta is also rich in oxygen nuclei. The numerical modeling of the early spectra and light curve of SN 1998bw (Nakamura et al. 2001) yields a composition of $M_{\mathrm{C}}: M_{\mathrm{O}}: M_{\mathrm{Ne}}: M_{\mathrm{Mg}}: M_{\mathrm{Si}}: M_{\mathrm{S}}: M_{\mathrm{Ca}}: M_{\mathrm{Fe}}=0.006: 0.71: 0.037: 0.034: 0.083: 0.041: 0.007: 0.09$. We show the dependence of $N_{\mathrm{CenA}}$ and $\langle A\rangle$ on $E_{p \text {,max }}$ in the WR stellar wind and hypernova ejecta composition scenarios respectively in Figure 5. One can see that as long as $E_{p, \max }$ is larger than $10^{19} \mathrm{eV}$, which can be reached in the GRB and hypernova scenarios (Wang et al. 2007, 2008), both $N_{\mathrm{CenA}}$ and $\langle A>$ meet the requirements. It has been also shown that the spectrum and composition of UHECRs accelerated in the WR stellar wind or in the hypernova ejecta are compatible with the PAO's observations (Liu \& Wang 2012).

Both (long) GRBs and hypernovae are generally believed to trace star formation. Recent observations by the Hubble Space Telescope revealed triggered star formation occurred $\lesssim 10 \mathrm{Myr}$ ago in the inner filament of Cen A 
(Crockett et al. 2012), although Cen A belongs to elliptical galaxies, which usually have low star formation rates. Possible alternative sites are GRBs and hypernovae occurring in the Centaurus cluster, which lies behind Cen A at a distance of $\sim 50 \mathrm{Mpc}$. Since there are hundreds of galaxies in the Centaurus cluster, the star formation rate is much higher than that in Cen A, and as a result, the rate of GRB or hypernova is much higher. For a distance of $\sim 50 \mathrm{Mpc}$, intermediate-mass nuclei such as oxygen of $\gtrsim 60 \mathrm{EeV}$ will suffer from photo-disintegration during the propagation, so the chemical composition at the border of the Galaxy after propagation may be different from that at the sources (also see the discussion in Taylor et al. 2011). Figure 6 shows the propagated spectrum of oxygen nuclei from the Centaurus cluster. The integrated flux from $10^{19.75} \mathrm{eV}$ to $10^{19.95} \mathrm{eV}$ are about a factor of 0.15 of the initial one. Heavier nuclei at higher energies also suffer from severe attenuation, while helium nuclei and protons at lowerenergies $\left(\sim 10^{18} \mathrm{eV}-10^{19} \mathrm{eV}\right)$ are almost not affected. Taking into account such attenuation, therefore, leads to even stronger constraints on the element abundance of light particles at the sources, i.e. approximately reducing to 0.15 times the value obtained in the previous section, leading to the new constraint of $M_{\mathrm{H}} \lesssim 0.33 M_{\mathrm{O}}$ and $M_{\mathrm{He}} \lesssim 0.43 M_{\mathrm{O}}$. Even though this new constraint is harder to satisfy, one finds that the chemical compositions of WR stellar winds and hypernovae ejecta can still satisfy such a strict requirement.

In this paper, we study the origin of the observed excess of UHECRs in the direction of Cen A. First, by simulating the propagation of cosmic rays in the GMF, we find that the excess events can not be mainly composed of heavy nuclei like iron nuclei coming from the direction of Cen A, because the GMF has a significant influence on their trajectories and cause their apparent arrival direction to severely deviate from their original directions. Also, the excess events are not likely to be dominated by light particles such as helium nuclei or protons as the measurements of the elongation rate of air showers by PAO suggest a heavy or intermediate mass composition. We show that intermediate mass nuclei with nuclear charge $6 \lesssim Z \lesssim 10$ are good candidates of the main composition of the excess events. We also show that the composition of cosmic rays can be further constrained when the anisotropy of low-energy cosmic rays is considered. In order not to produce a significant anisotropy in the direction of Cen A at lower energies, which is not observed by the PAO, a low proton and helium abundance (e.g. a supersolar metallicity) is required. None of the proposed candidate acceleration sites in Cen A is favored by this constraint. We find that the cosmic-ray accelerators arising from stellar explosions such as GRBs or hypernovae are more favorable sources of the excess events because high abundance of intermediate mass elements could be possible in these accelerators. Since the event rates of GRBs or hypernovae trace the star formation rate, the star formation region in the inner filament of Cen A or in the Centaurus Cluster may be the origin of the excess events. However, as a word of caution, it should be remembered that the excess is significant only a posteriori. Furthermore we note that due to uncertainties in our knowledge of the hadronic model describing cosmic ray atmospheric showers, the GMFs, the chemical environment of Cen A and even the acceleration mechanism of UHECRs, the conclusions made in this paper should be regarded as model-dependent.

We thank Gwenael Giacinti, Zhuo Li and Hasan Yüksel for valuable comments, Xiao-Hui Sun, Yun-Ying Jiang and Li-Gang Hou for useful discussions on the GMF, Jakub Vicha, Lu Lu and Tao Wang for helps in understanding the observations of the PAO, Feng Chen for help in parallel computation. The numerical calculations in this paper have been done on the IBM Blade cluster system in the High Performance Computing Center (HPCC) of Nanjing University. This work is supported by the NSFC under grants 10973008 and 11033002, the 973 program under grant 2009CB824800, the program of NCET, and the Fok Ying Tung Education Foundation. LRY is grateful to all the members in the group leaded by Jin-Lin Han in NAOC for their friendly hospitality and creative atmosphere.

\section{REFERENCES}

Abbasi, R. U., Abu-Zayyad, T., Amann, J. F., et al. 2004, Physical Review Letters, 92, 151101 
Abbasi, R. U., Abu-Zayyad, T., Al-Seady, M., et al. 2010, Physical Review Letters, 104, 161101

Abdo, A. A., Ackermann, M., Ajello, M., et al. 2010, Science, 328, 725

Abraham, J., Abreu, P., Aglietta, M., et al. 2010, Physical Review Letters, 104, 091101

Abraham, J., Abreu, P., Aglietta, M., et al. 2010, Physics Letters B, 685, 239

Anchordoqui, L. A., Goldberg, H., \& Weiler, T. J. 2001, Physical Review Letters, 87, 081101

Arav, N., Gabel, J. R., Korista, K. T., et al. 2007, ApJ, 658, 829

Barai, P., Martel, H., \& Germain, J. 2011, ApJ, 727, 54

Berezinsky, V., Gazizov, A., \& Grigorieva, S. 2006, Phys. Rev. D, 74, 043005

Bieging, J. H. 1990, The Evolution of the Interstellar Medium, 12, 137

Boldt, E., \& Ghosh, P. 1999, MNRAS, 307, 491

Cavallo, G. 1978, A\&A, 65, 415

Chiosi, C., \& Maeder, A. 1986, ARA\&A, 24, 329

Crockett, R. M., Shabala, S. S., Kaviraj, S., et al. 2012,arXiv:1201.3369

Crowther P. A. 2007, ARA\&A, 45, 177

Dermer, C. D., Razzaque, S., Finke, J. D., \& Atoyan, A. 2009, New Journal of Physics, 11, 065016

Ellison, D. C., Baring, M. G., \& Jones, F. C. 1996, ApJ, 473, 1029

Fargion, D. 2011, Nuclear Instruments and Methods in Physics Research A, 630, 111

Fields, D. L., Mathur, S., Krongold, Y., Williams, R., \& Nicastro, F. 2007, ApJ, 666, 828

Giacinti, G., Kachelrieß, M., Semikoz, D. V., \& Sigl, G. 2010, JCAP, 8, 36

Giacinti, G., Kachelrieß, M., Semikoz, D. V., \& Sigl, G. 2011, Astroparticle Physics, 35, 192

Gureev, S., \& Troitsky, S. 2010, International Journal of Modern Physics A, 25, 2917

Han, J. L., \& Qiao, G. J. 1994, A\&A, 288, 759

Han, J. L., Manchester, R. N., \& Qiao, G. J. 1999, MNRAS, 306, 371

Han, J. L., Manchester, R. N., Berkhuijsen, E. M., \& Beck, R. 1997, A\&A, 322, 98

Han, J. L., Manchester, R. N., Lyne, A. G., Qiao, G. J., \& van Straten, W. 2006, ApJ, 642, 868

Harari, D., Mollerach, S., \& Roulet, E. 1999, Journal of High Energy Physics, 8, 22

Harari, D., Mollerach, S., \& Roulet, E. 2000, Journal of High Energy Physics, 2, 35

Harari, D., Mollerach, S., Roulet, E., \& Sánchez, F. 2002, Journal of High Energy Physics, 3, 45

Hardcastle, M. J., Cheung, C. C., Feain, I. J., \& Stawarz, Ł. 2009, MNRAS, 393, 1041 
Harris, G. L. H., Rejkuba, M., \& Harris, W. E. 2010, PASA, 27, 457

Honda, M. 2009, ApJ, 706, 1517

Hlavacek-Larrondo, J., Fabian, A. C., Sanders, J. S., \& Taylor, G. B. 2011, MNRAS, 415, 3520

Hooper, D., \& Taylor, A. M. 2010, Astroparticle Physics, 33, 151

Hou, L. G., Han, J. L., \& Shi, W. B. 2009, A\&A, 499, 473

Jiang, Y.-Y., Hou, L. G., Han, J. L., Sun, X. H., \& Wang, W. 2010, ApJ, 719, 459

Kim, D.-W. 2012, Astrophysics and Space Science Library, 378, 121

Kraft, R. P., Forman, W. R., Hardcastle, M. J., et al. 2009, ApJ, 698, 2036

Lemoine, M., \& Waxman, E. 2009, jcap, 11, 9

Liu, R.-Y., \& Wang, X.-Y. 2012, ApJ, 746, 40

Lodders, K., \& Palme, H. 2009, Meteoritics and Planetary Science Supplement, 72, 5154

Mannheim, K. 1995, Astroparticle Physics, 3, 295

Moll, R., Schindler, S., Domainko, W., et al. 2007, A\&A, 463, 513

Morris, M., \& Serabyn, E. 1996, ARA\&A, 34, 645

Nakamura, T., Mazzali, P. A., Nomoto, K., \& Iwamoto, K. 2001, ApJ, 550, 991

Neronov, A. Y., Semikoz, D. V., \& Tkachev, I. I. 2009, New Journal of Physics, 11, 065015

Nota, T., \& Katgert, P. 2010, A\&A, 513, A65

O’Sullivan, S., Reville, B., \& Taylor, A. M. 2009, MNRAS, 400, 248

Paczyński, B. 1998, Gamma-Ray Bursts, 4th Hunstville Symposium, 428, 783

Panov, A. D., Adams, J. H., Jr., Ahn, H. S., et al. 2007, Bulletin of the Russian Academy of Science, Phys., 71, 494

Panov, A. D., Adams, J. H., Ahn, H. S., et al. 2009, Bulletin of the Russian Academy of Science, Phys., 73, 564

Pe'er, A., \& Loeb, A. 2011, arXiv:1111.3964

Prouza, M., \& Šmída, R. 2003, A\&A, 410, 1

Richter, P., Charlton, J. C., Fangano, A. P. M., Bekhti, N. B., \& Masiero, J. R. 2009, ApJ, 695, 1631

Rieger, F. M., \& Aharonian, F. A. 2009, A\&A, 506, L41

Romero, G. E., Combi, J. A., Perez Bergliaffa, S. E.,

Tinyakov, P. G., \& Tkachev, I. I. 2005, Astroparticle Physics, 24, 32

Schmidt, R. W., Fabian, A. C., \& Sanders, J. S. 2002, MNRAS, 337, 71

Shaham, N., \& Piran, T. 2012, arXiv:1204.1488 
Sommers, P. 2001, Astroparticle Physics, 14, 271

Stanev, T. 1997, ApJ, 479, 290

Taylor, A. M., Ahlers, M., \& Aharonian, F. A. 2011, Phys. Rev. D, 84, 105007

Taylor, A. M, the lecture given by A.M. Taylor at Dublin summer school on high energy astrophysics, 2011 http://homepages.dias.ie/ cappa/work_shop/Andrew_Taylor1.pdf

The Pierre Auger Collaboration, et al. 2007, Science, 318, 938

The Pierre AUGER Collaboration, et al. 2008, Astroparticle Physics, 29, 188

The Pierre AUGER Collaboration, et al. 2010, Astroparticle Physics, 34, 314

Pierre Auger Collaboration, Abreu, P., Aglietta, M., et al. 2011, JCAP, 6, 22

The Pierre Auger Collaboration, Abreu, P., Aglietta, M., et al. 2011, arXiv:1107.4804

Tinyakov, P. G., \& Tkachev, I. I. 2002, Astroparticle Physics, 18, 165

Tinyakov, P. G., \& Tkachev, I. I. 2005, Astroparticle Physics, 24, 32

Tsunesada, Y. for the Telescope Array Collaboration 2011, arXiv:1111.2507

Pignata, G., et al. 2009, Central Bureau Electronic Telegrams, 1731, 1

Piran, T. 2010,arXiv:1005.3311

Ulrich, R., Engel, R., Müller, S., et al. 2009, arXiv:0906.0418

van der Hucht, K. A., Cassinelli, J. P., \& Williams, P. M. 1986, A\&A, 168, 111

Wang, X.-Y., Razzaque, S., Mészáros, P., \& Dai, Z.-G. 2007, Phys. Rev. D, 76, 083009

Wang, X.-Y., Razzaque, S., \& Mészáros, P. 2008, ApJ, 677, 432

Waxman, E. 1995, Physical Review Letters, 75, 386

Waxman, E. 2011, arXiv:1101.1155

Yüksel, H., Stanev, T., Kistler, M. D., \& Kronberg, P. P. 2012, arXiv:1203.3197

Yusef-Zadeh, F., Morris, M., \& Chance, D. 1984, Nature, 310, 557

Yusef-Zadeh, F., Hewitt, J. W., \& Cotton, W. 2004, ApJS, 155, 421 
Table 1: Some useful parameters obtained in our simulation. $\bar{\zeta}$ is the averaged number ratio of particles that originate from the direction of Cen A and reach the Earth when the GMF deflection is considered to those that reach the Earth without considering the GMF deflection, $\bar{\eta}$ is the averaged number ratio of particles that reach the Earth from a direction within $18^{\circ}$ of Cen A to those that reach the Earth from all directions, and $N_{\text {CenA }}$ is the maximum event number of cosmic rays that are confined within $18^{\circ}$ of Cen A out of 64 UHECR events.

\begin{tabular}{ccccccc}
\hline \hline & \multicolumn{3}{c}{ PS model } & \multicolumn{3}{c}{ J model } \\
Elements & $\bar{\zeta}$ & $\bar{\eta}$ & $N_{\text {CenA }}$ & $\bar{\zeta}$ & $\bar{\eta}$ & $N_{\text {CenA }}$ \\
\hline $\mathrm{H}$ & 1.00 & 0.96 & 27.8 & 0.95 & 1 & 13 \\
$\mathrm{He}$ & 1.03 & 0.93 & 27.0 & 0.95 & 1 & 13 \\
$\mathrm{O}$ & 2.12 & 0.41 & 11.9 & 1.84 & 0.66 & 20.5 \\
$\mathrm{Si}$ & 1.80 & 0.02 & 0.58 & 2.55 & 0.25 & 8.00 \\
$\mathrm{Ca}$ & 0.86 & 0.008 & 0.23 & 0.79 & 0.07 & 2.31 \\
$\mathrm{Fe}$ & 0.88 & 0.01 & 0.33 & 0.44 & 0.004 & 0.13 \\
\hline
\end{tabular}



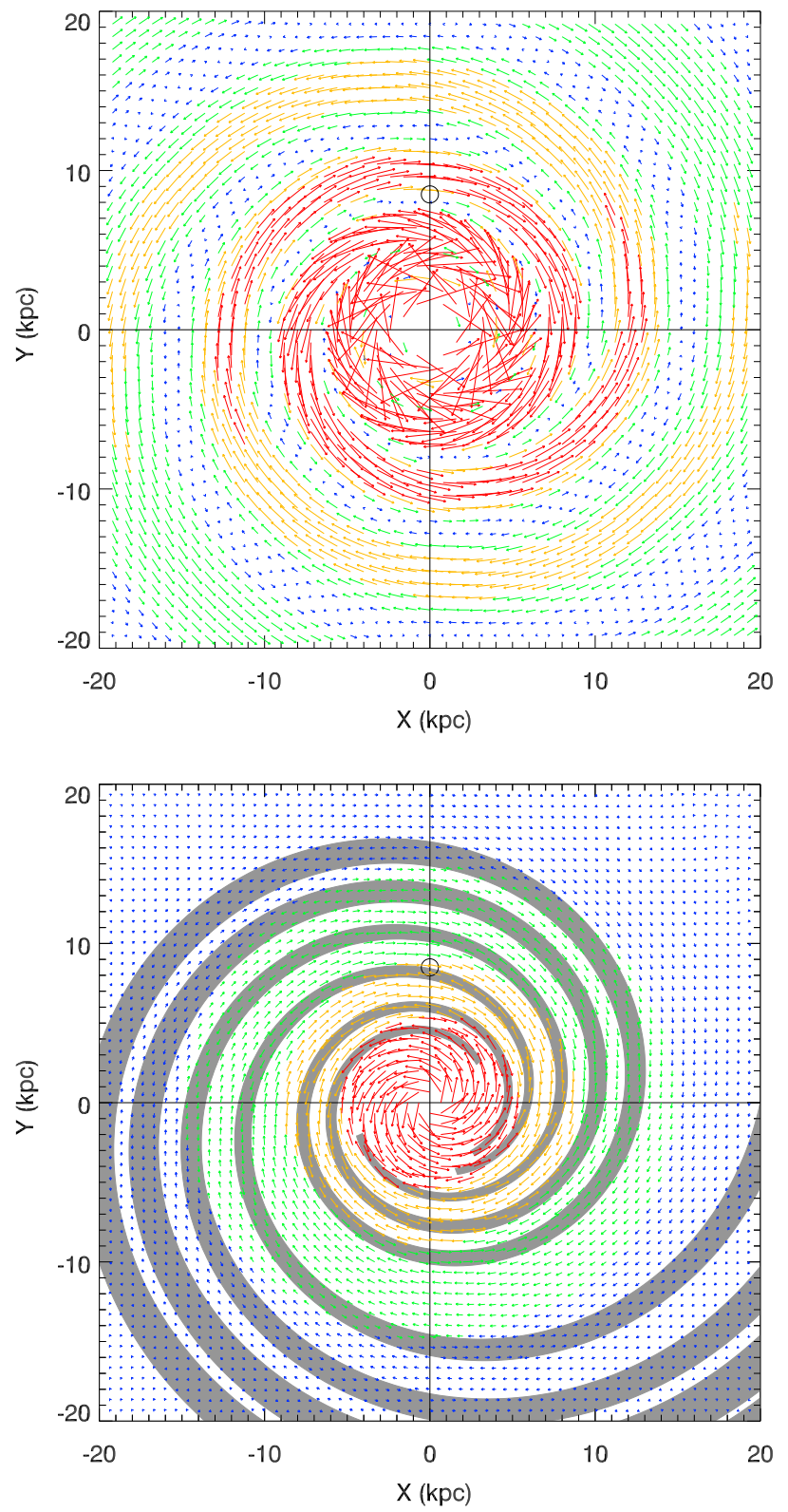

Fig. 1. - Configuration of the disk magnetic field in the PS model (upper panel) and the $\mathbf{J}$ model (bottom panel), respectively. The direction of arrows represents the direction of the field while the length of arrows represents the strength of the field. In particular, magnetic field $>3 \mu \mathrm{G}$ is colored as red, $>2 \mu \mathrm{G}$ yellow, $>1 \mu \mathrm{G}$ green, $<1 \mu \mathrm{G}$ blue. The open circle shows the location of the sun. For clarity, the field at the Galactic center is not shown. The shaded region in the bottom panel outlines four spiral arms. 

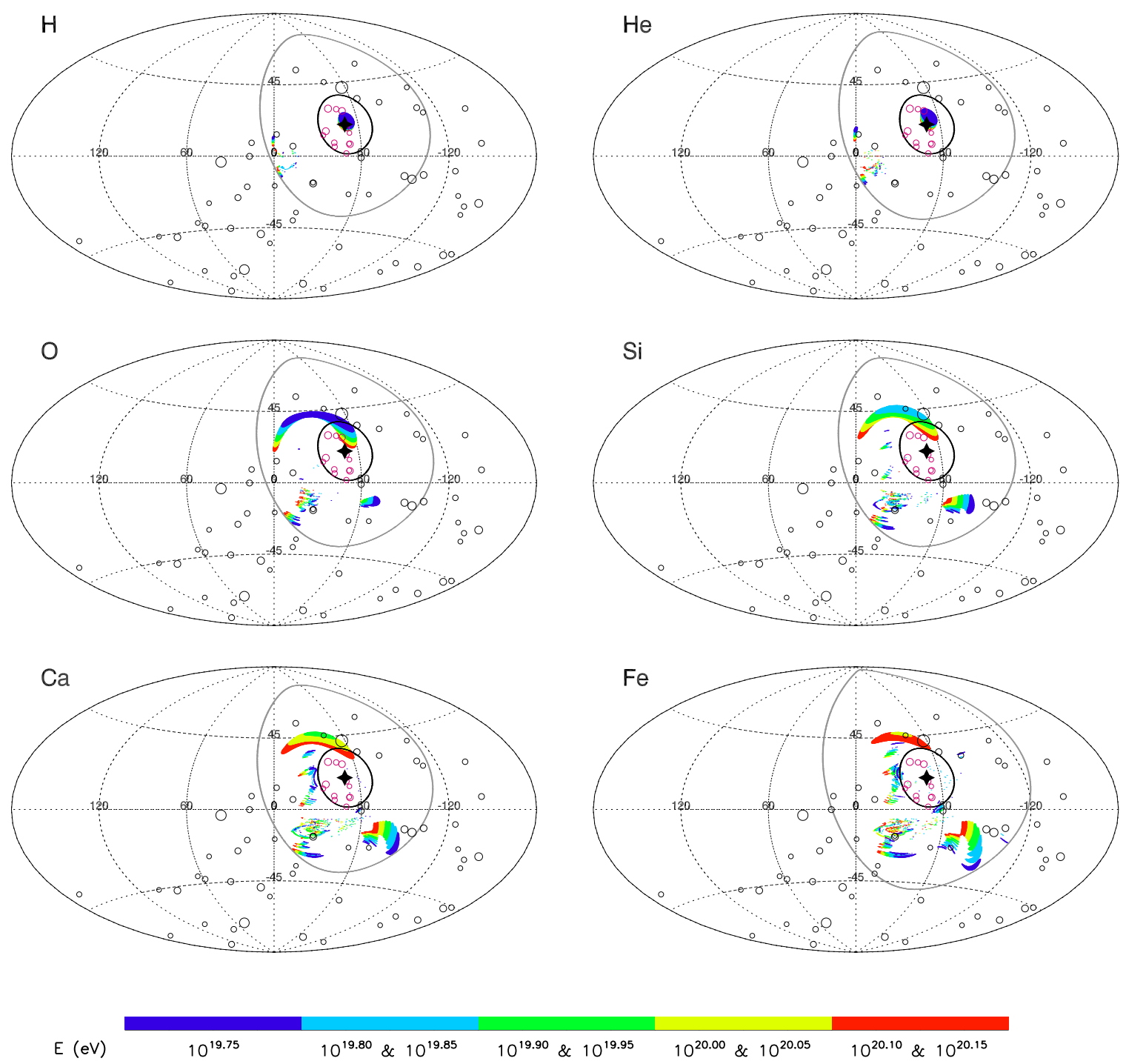

Fig. 2.- Arrival directions (in Galactic coordinates) of cosmic rays originating from the direction of Cen A in the PS model. A total of $10^{6}$ particles are used in these simulations. One dot represents one particle, and different color dots/bands represent different energies (from $10^{19.75} \mathrm{eV}$ to $10^{20.15} \mathrm{eV}$ with an increasement of $10^{0.05}$ ). The black filled star is the location of Cen A $\left(l=309.52^{\circ}, b=19.42^{\circ}\right)$ and the black solid curve is the projection on the celestial sphere of a circle centered at Cen A with a radius of $18^{\circ}$. The pale solid curve is the projection of a circle centered at Cen $\mathrm{A}$ with a radius equal to the largest deflection angle of arriving cosmic rays. The small open circles represent the observed events and the size of the circle is proportional to the energy. The 13 magenta ones are those events that are correlated with Cen A. See text for more discussions. 

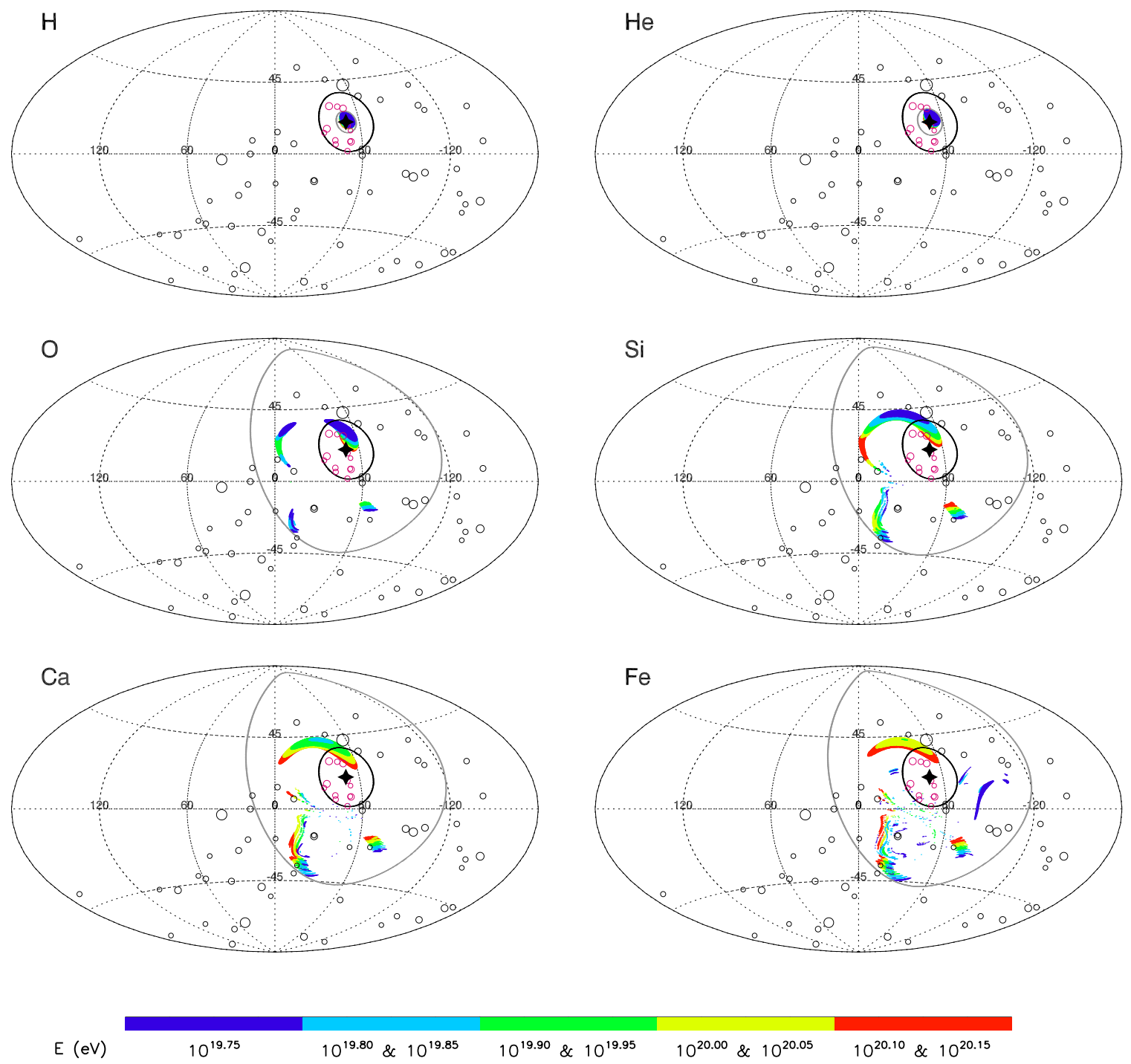

Fig. 3. - The same as Figure 2 but for the case of the J model. See text for more discussions. 


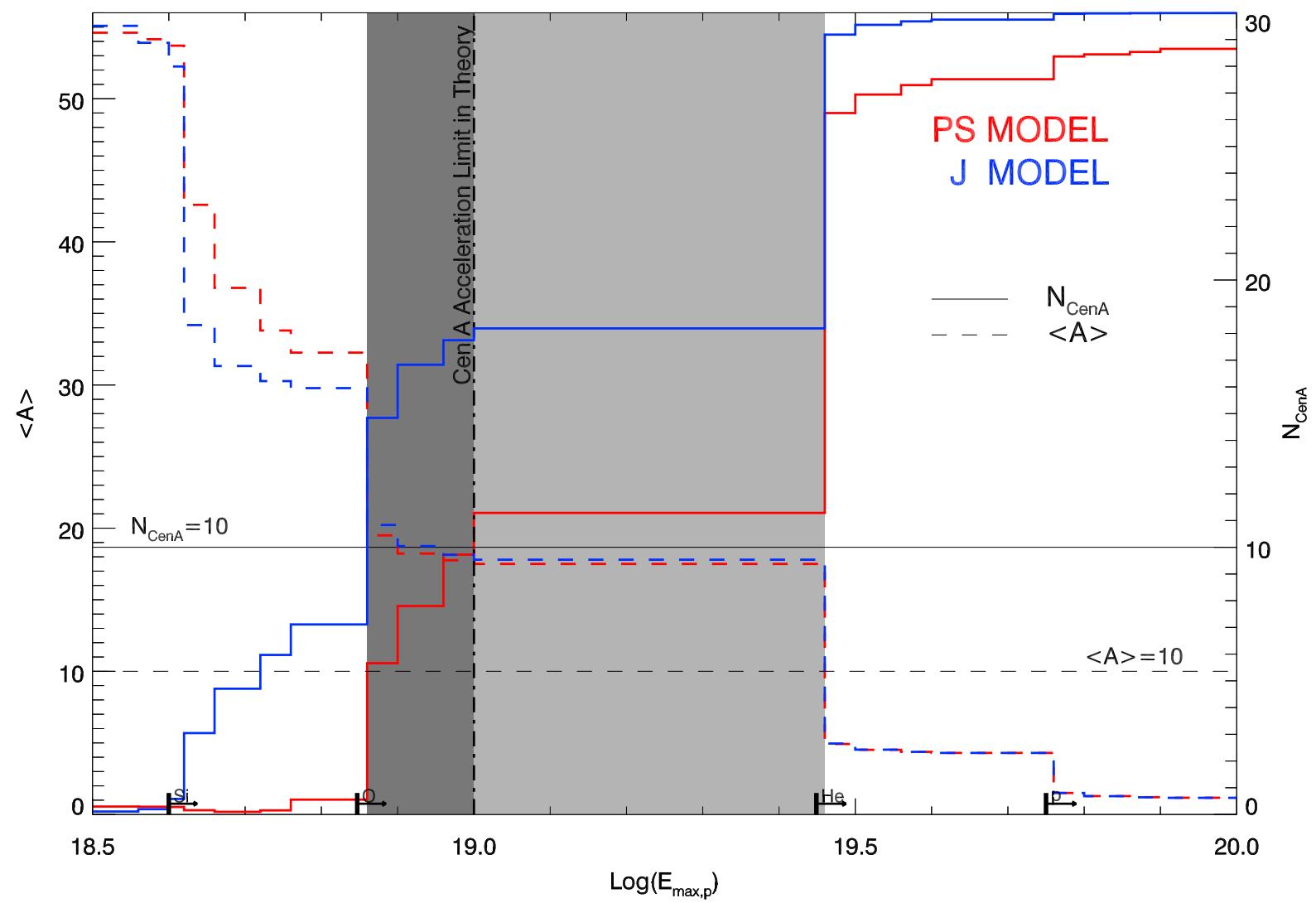

Fig. 4. - The maximum number of cosmic rays $N_{\text {cenA }}$ from the direction of Cen A and the average atom mass $<A>$ versus different maximum proton acceleration energy $E_{p, \max }$ for solar abundance composition of cosmic rays. The average atom mass $\langle A\rangle$ is shown by the dashed lines with the value indicated by the left-handed vertical axis, while $N_{\text {cenA }}$ is shown by the solid lines with the value indicated by the right-handed vertical axis. The corresponding values of $E_{p, \max }$ where "Si", "O" and etc are indicated are the lower limits of $E_{p, \max }$ for which nuclei of such elements can be accelerated to $10^{19.75} \mathrm{eV}$. The shaded region represents the appropriate range of $E_{p, \max }$ that satisfy both $N_{\text {cenA }} \gtrsim 10$ and $\langle A\rangle \gtrsim 10$. The vertical dash-dotted line shows an approximate theoretical acceleration upper limit for protons in Cen A ( $\sim 10^{19} \mathrm{eV}$, e.g. Hardcastle et al. 2009; Lemoine \& Waxman 2009; Piran 2010; Gureev \& Troitsky 2010), however, which is still under much debate. See text for more discussion. 

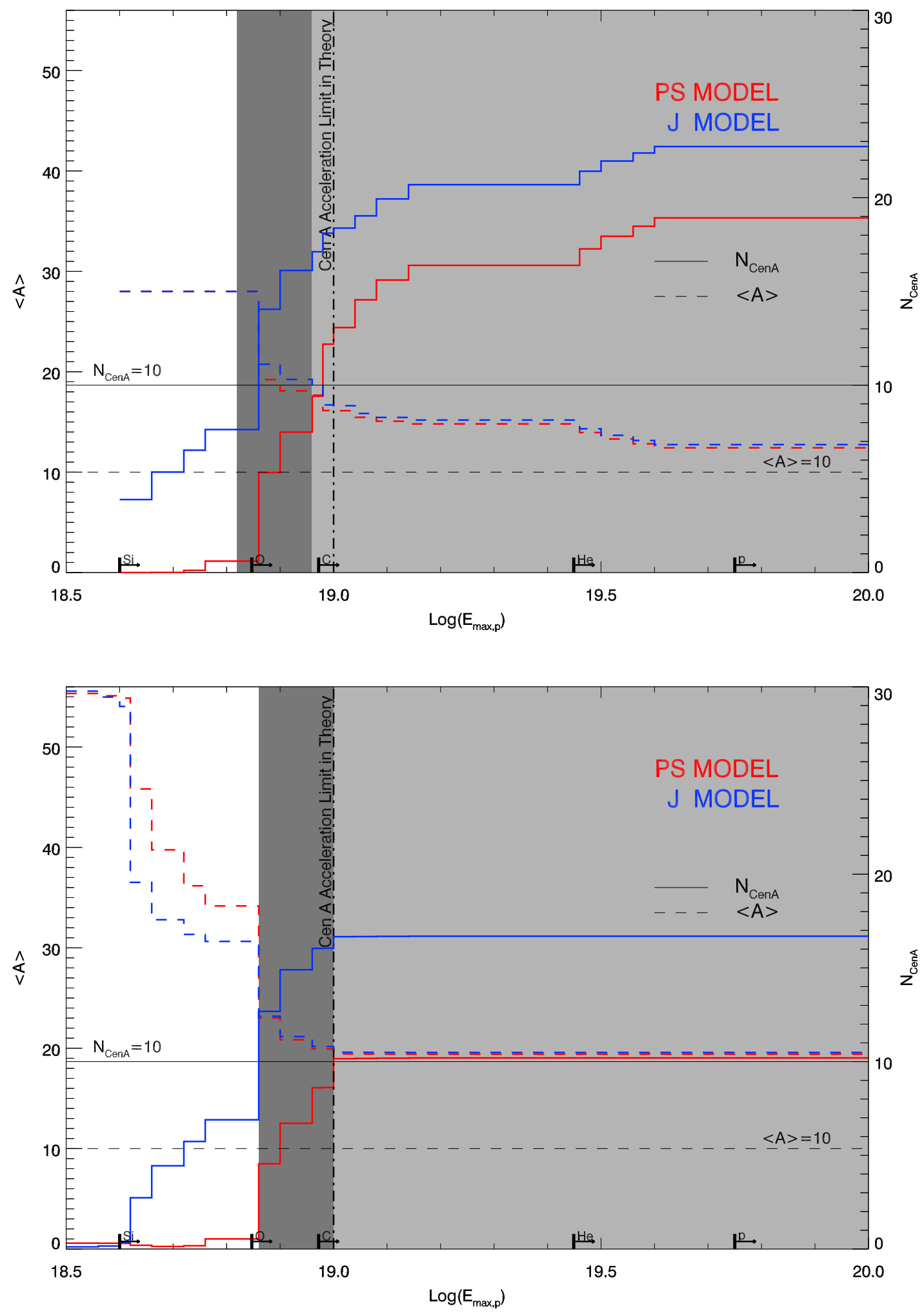

Fig. 5.- The same as Figure 4 but for different cosmic-ray composition. The top panel corresponds to a WR stellar wind composition and the bottom panel corresponds to the composition of hypernova ejecta. See text for more discussions. 


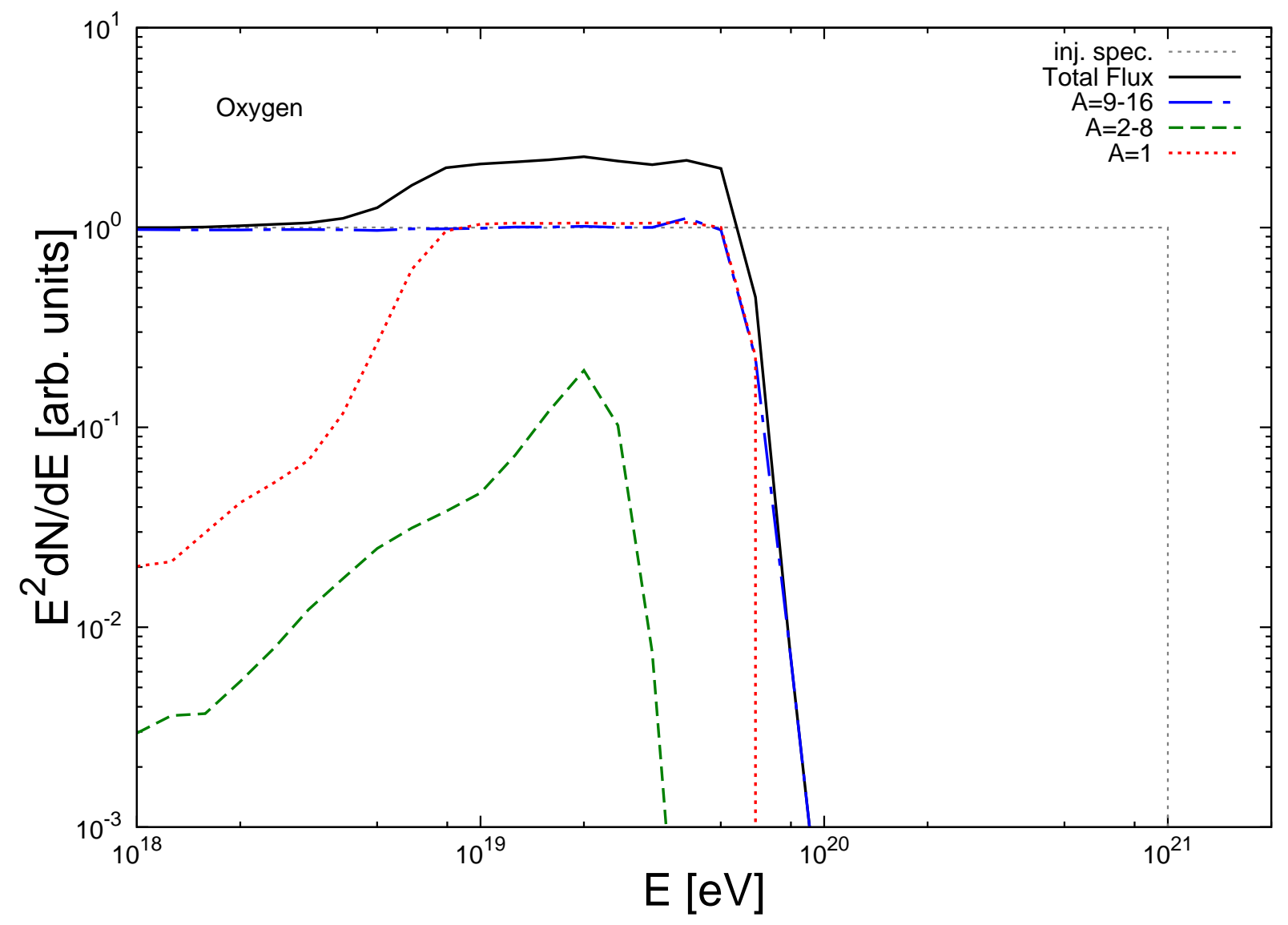

Fig. 6.- The propagated spectrum of oxygen nuclei after traveling a distance of $50 \mathrm{Mpc}$. The initial spectrum is set to be a power-law spectrum with index of -2 and with an abrupt cutoff at $10^{21} \mathrm{eV}$. 